\title{
Prandtl number dependence of convective fluids in tall laterally heated slots
}

\author{
J. Sánchez Umbría ${ }^{\mathrm{a}}$ and M. Net ${ }^{\mathrm{b}}$ \\ Departament de Física, Universitat Politècnica de Catalunya
}

\begin{abstract}
The influence of the Prandtl number on the stable stationary and periodic flows of the fluids contained in laterally heated slots under realistic conditions, namely non-slip boundaries, insulated top and bottom horizontal limits and perfectly conducting lateral sides, is analyzed by using continuation methods. The branches of solutions are computed by decreasing the Prandtl number, for four Rayleigh numbers. The dynamical behavior depends strongly on both parameters. For a Rayleigh number, $\mathrm{Ra}=10^{3}$, the steady flow remains stable in the wide range of Prandtl numbers computed. At $\mathrm{Ra}=10^{4}$ and $\mathcal{O}\left(10^{5}\right)$ the first bifurcations are of Hopf type giving rise to a type of oscillations that affects the bulk of the fluid, alternating from a general circulation to multi-vortex solutions, or the boundary layer, respectively. However, it is found that, in any case, the location of the shear determines the type of the oscillations. Moreover, at $\mathrm{Ra}=10^{4}$ the critical multipliers at the secondary bifurcations on the main branch of POs are real, giving rise to different kinds of very bounded stable periodic states of different symmetries and periods. At Ra of order $10^{5}$ the instability of the periodic orbits gives rise directly to quasi-periodic flows.
\end{abstract}

\section{Introduction}

The knowledge of thermal convection in Cartesian geometries heated by the side is important for several industrial applications as for the successful growth of crystals in liquid media [1], the design of large-scale laser systems [2] or the optimal heating or cooling and isolation of buildings. Their systematic study started in the seventies, but it is from the nineties when this problem has been intensively analyzed. A detailed review of the bibliography up to the last decade can be found in Ref. [3]. With the diffusion of continuation methods for steady flows and travelling waves in large scale dissipative systems $[4,5]$, most of the research focused on their dynamics and stability (see for instance Ref. [6-10]), but now, with the development of continuation methods for periodic orbits [11-13], it is possible to go deeper into the study of the periodic dynamics at the same level than for the stationary flows.

Laterally heated gases ( $\mathrm{Pr}$ order one) in tall closed cavities with adiabatic horizontal walls first develop a basic stable flow consisting of a single steady vortex centered in the domain. This flow is center-symmetric. The type of instability of

\footnotetext{
a e-mail: juan.j.sanchez@upc.edu

b e-mail: marta.net@upc. edu
} 
this flow depends strongly on the ratio between the thermal and the viscous time scales, i.e. on the Prandtl number, Pr, and the aspect ratio. The numerical simulations of S. Wakitani [14], among others, showed that in very tall containers (aspect ratio $\Gamma=h / d \gtrsim 11, h$ being the height of the cavity, and $d$ its width), filled with air of $\operatorname{Pr}=0.71$, the general circulation is superseded by multi-vortex steady states when $\mathrm{Ra}$ is increased. This parameter gives the ratio between the work done by the buoyancy force, and the energies dissipated by the viscosities. Then, the steady flows lose stability giving rise to multi-vortex oscillations. The study of the transition from the steady to oscillatory flows for $\operatorname{Pr}=0.71$ and $7 \lesssim \Gamma \lesssim 11$ showed the coexistence of various branches of periodic orbits consisting of waves travelling along the boundary layer, and a stable core of fluid almost horizontally stratified $[15,8,16,17]$, consequently the global circulation is maintained.

The dependence on $\Gamma$ of the steady and oscillatory flows in slots, for air $(\operatorname{Pr}=0.71)$ and liquid metals ( $\operatorname{Pr}=0.025)$, has been studied by many authors. However, in between, there is a gap of $\operatorname{Pr}$ not covered before. This study tries to fill this gap by examine the origin and behavior of fluids of intermediate Prandtl numbers. New computations by using continuation methods with Pr as continuation parameter, and stability analysis, are presented. As in [17], a rectangular domain of $\Gamma=8$ is taken because it is tall enough as to allow to capture the dynamics of the convection in very tall systems. In the range $0.2<\operatorname{Pr}<0.3$ time periodic solutions, not described before in the literature, consisting in transients between a time global circulation filling the slot and the formation of isolated vortices have been found. Although the problem considered is two-dimensional it is relevant because the onset of time-dependent flows is two-dimensional for boxes of large aspect ratios if there is enough space in the periodic transverse direction, and the orders of magnitude of the heat flux and kinetic energy averages calculated in this study should not differ very much from those of the three-dimensional problem. Its knowledge is significant, for instance for the working of binary gaseous mixtures in thermoacoustic engines [18]. According to this study, the better working gases are mixtures of helium and xenon that can reach low Prantl numbers and the highest ratio of specific heats at pressures lower than 4.5 MPa. The thermal penetration depth is an important factor to take into account in the design of this type of engines.

The paper is organized as follows. The mathematical model is summarized in Sec. 2, after the introduction. Secs. 3 and 4 contain the results obtained for the steady states (SSs) and the periodic orbits (POs) bifurcated from the steady branch, respectively. They include the study of the origin of the instabilities of the steady flows and the dynamics of those periodic. The paper finishes in Sec. 5 with some conclusions, and a summary of the main results obtained.

\section{Mathematical formulation}

The thermal convection of fluids of $\mathrm{Pr}$ ranging from 0.683 to 0.014 , filling a rectangular slot, $\Omega$, is studied. The boundaries are taken non-slip, and the lateral sides are maintained at uniform temperatures, the left higher than the right. The top and bottom contours are insulating, and the fluid is subject to a vertical gravity $\mathbf{g}=-g \mathbf{j}$, j being the unit vector pointing upwards.

The problem is formulated as in Ref. [17]. The Boussinesq approximation of the Navier-Stokes and temperature equations in terms of the stream function, $\psi$, and the deviation of the temperature, $\Theta$, is used. The stream function is related to the velocity field by $(u, v)=\left(\partial_{y} \psi,-\partial_{x} \psi\right)$. These equations are nondimensionalized by taking $d$, the difference of temperature $\Delta \mathcal{T}>0$ between the left and right sides, and $d^{2} / \kappa, \kappa$ being the thermal diffusivity, as distance, temperature and thermal time 
scales, respectively. In non-dimensional units $\Omega=[0,1] \times[0, \Gamma]$. With that the system solved is

$$
\begin{aligned}
& \partial_{t} \Delta \psi-J(\psi, \Delta \psi)=\operatorname{Pr} \Delta^{2} \psi+\operatorname{Ra} \operatorname{Pr}\left(1-\partial_{x} \Theta\right), \\
& \partial_{t} \Theta-J(\psi, \Theta)=\Delta \Theta+\partial_{y} \psi,
\end{aligned}
$$

with $J(f, g)=\partial_{x} f \partial_{y} g-\partial_{y} f \partial_{x} g$. The deviation of the temperature $\Theta$ comes from the splitting the full temperature as $\mathrm{T}(x, y, t)=(1-x)+\Theta(x, y, t)$, in order of having homogeneous boundary conditions on the vertical walls. The symbols $x$ and $y$ account for the horizontal and vertical coordinates, respectively.

The boundary conditions are

$$
\begin{aligned}
& \psi=\partial_{x} \psi=0 \text { on } y=0, \Gamma, \text { and } \psi=\partial_{y} \psi=0 \text { on } x=0,1 \\
& \partial_{y} \Theta=0 \text { on } y=0, \Gamma \text { and } \Theta=0 \text { on } x=0,1 .
\end{aligned}
$$

The problem depends on the Rayleigh and Prandtl numbers defined as

$$
\mathrm{Ra}=\frac{\alpha g \Delta \mathrm{T} d^{3}}{\kappa \nu} \quad \text { and } \quad \operatorname{Pr}=\frac{\nu}{\kappa},
$$

where $\alpha$ is the thermal expansion coefficient, $\nu$ the kinematic viscosity, $\kappa$ the thermal diffusivity. The second will be used as control parameter.

System (1)-(4) is $\mathcal{Z}_{2}$ equivariant. It remains invariant under the center-symmetry

$$
\mathcal{S}:(t, x, y, \psi, \Theta) \rightarrow(t, 1-x, \Gamma-y, \psi,-\Theta) .
$$

The functions $\psi$, and $\Theta$ are approximated by a pseudo-spectral collocation method on a stretched mesh of $n_{x} \times n_{y}=40 \times 140$ Gauss-Lobatto points, which accumulate at the boundaries of the domain. It was checked that this number of points is enough to solve even the thin boundary layers of the convective flows of fluids of $\operatorname{Pr}=0.7$. This study focuses on Prandtl numbers lower than that, for which the boundary layers are wider. The stiff system of ODEs obtained after the spatial discretization is integrated by means of five-order semi-implicit BDF-extrapolation formulas. To compute branches of steady and time periodic solutions Newton-Krylov methods are used. The linear systems are solved using GMRES, and to compute the stability ARPACK is employed. The matrix-matrix products are calculated by means of the BLAS library. Additional details about the resolution of the problem can be found in Ref. [19].

\section{Steady flows and their stability}

The branches of the steady solutions and their stability have been computed from $\operatorname{Pr}=0.683$ by decreasing the continuation parameter. Fig. 1 shows these branches and the POs bifurcated from the point where the former lose stability, for $\mathrm{Ra}=10^{3}$, $\mathrm{Ra}=10^{4}, \mathrm{Ra}=10^{5}$ and $\mathrm{Ra}=3 \times 10^{5}$, as a function of Pr. The vertical axis represents the averaged heat flux at the section $x=1$, defined as

$$
\left[\overline{\partial_{x} \mathrm{~T}}\right]=\frac{1}{T \Gamma} \int_{0}^{T} \int_{0}^{\Gamma} \partial_{x} \mathrm{~T} d y d t .
$$

The time average concerns only the POs.

The shape of the curves of SSs changes a lot by changing Ra. At low Ra they are smooth in the range of Pr calculated. See for instance Figs. 1a, b for $\mathrm{Ra}=10^{3}$ and 

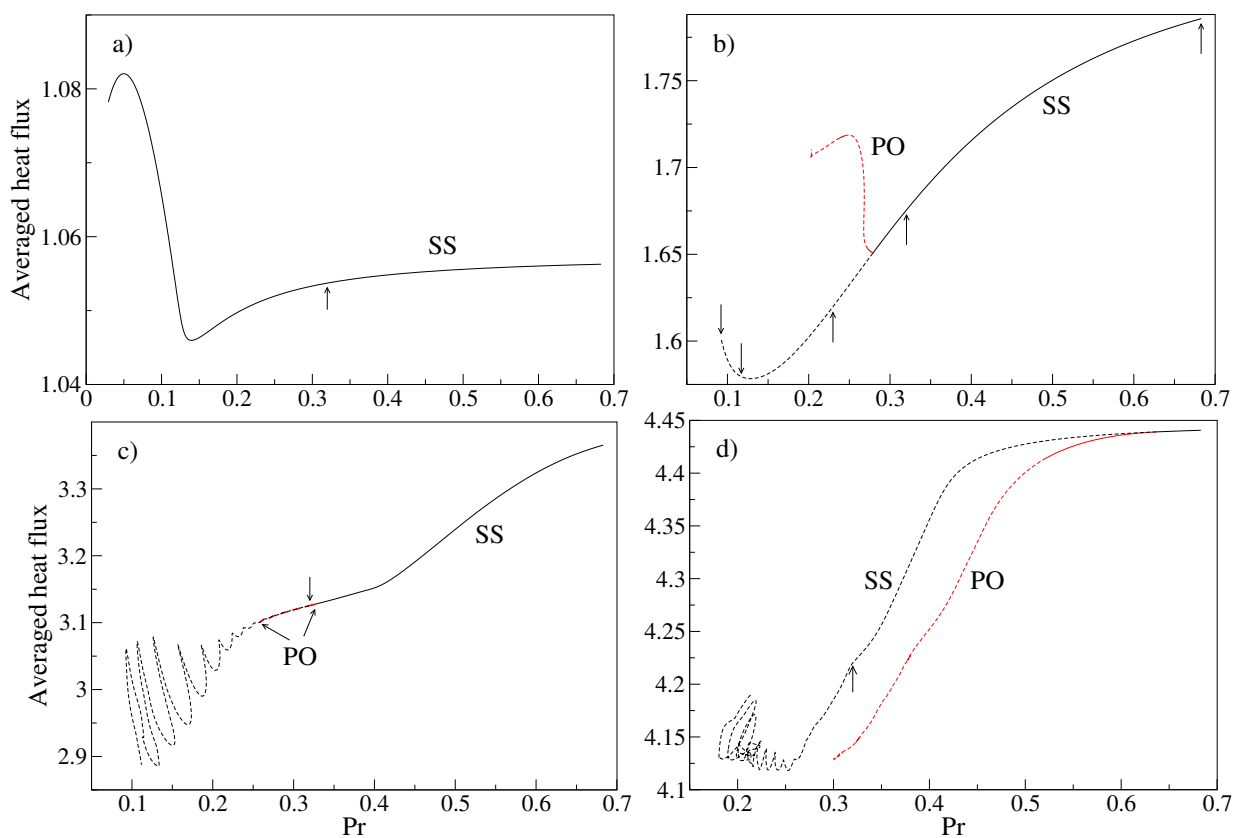

Fig. 1. Bifurcation diagrams showing branches of SSs and POs for a) $R a=10^{3}$, b) $R a=10^{4}$, c) $\mathrm{Ra}=10^{5}$ and d) $\mathrm{Ra}=3 \times 10^{5}$. The averaged heat transport versus $\mathrm{Pr}$ is depicted. Solid lines mean stable solutions while dashed lines indicate those that are unstable. Online POs are depicted in red. The arrows mark the position of the solutions shown in Figs. 2 and 3.

$\mathrm{Ra}=10^{4}$, respectively. They only have a minimum at $\operatorname{Pr}=0.1401$ and $\operatorname{Pr}=0.1286$, respectively. This point corresponds to the starting of a decrease of the mean kinetic energy (not shown here), that, at least in the first case, continues after the maximum of the curve of Fig. 1a and the following decrease of $\left[\overline{\partial_{x} T}\right]$. At low Pr and high Ra they look much more involved. At $\operatorname{Ra}=\mathcal{O}\left(10^{5}\right)$ and $\operatorname{Pr}<0.3$ (see Figs. 1c and d) the curves become very wavy and finally develop several cusps and loops of increasing amplitude when Pr is decreased. There are several eigenvalues of positive real part at the final point computed, although the largest is only $\mathcal{O}(1)$.

The patterns of the steady flows depend on both Ra and Pr. At large Ra and Pr of order one the flow consists of a single stationary vortex filling the domain with narrow vertical boundary layers. The fluid goes up near the hot side and down along the cold. In the central region of the slot the horizontal temperature gradient is very weak. By fixing $\mathrm{Pr}$, the effect of decreasing $\mathrm{Ra}$ is to confine the big vortex to the center of the slot. Fig. 2, shows the velocity field (black arrows) superposed to the contour plots of the full temperature, $\mathrm{T}$, for $\operatorname{Pr} \approx 0.32$. Since the solutions are stationary, the arrows of the velocity field are tangent to the contour plots of $\psi$, and indicate the trajectories of the particles of fluid. New initially weak vortices appear near the lower left and upper right corners (Fig. 2a). When Ra becomes smaller, the extra vortices intensify as in Fig. 2b, and after they weaken and move to the center (Fig. 2c) reducing the size of the central one. However, as can be seen in Fig. 2d, at $\mathrm{Ra}=10^{3}$ the extra vortices have not yet appeared, and lower values of Pr are needed to observe them. At very low Pr they become as strong as the initial. Moreover, the contour plots of the temperature show that the stronger interior horizontal velocity field injects hot fluid to the cold region and cold to the hot at different levels (depending on the position of the external vortices), tending to undulate the contour plots of the 

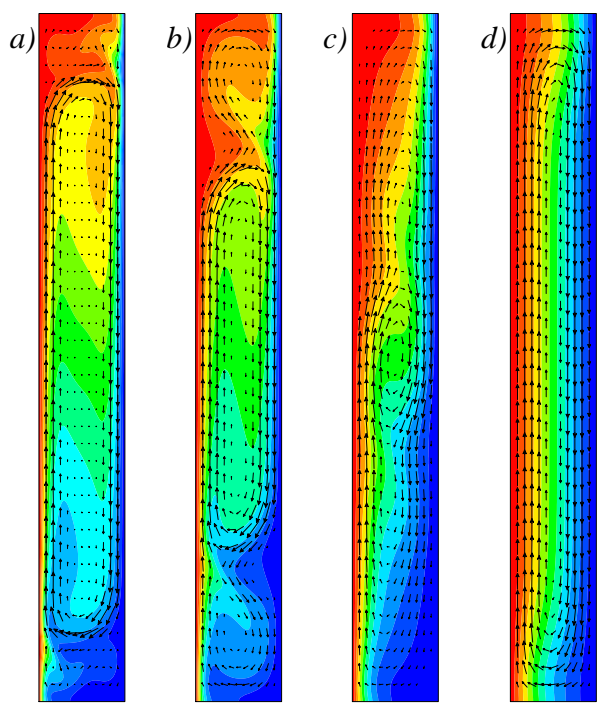

Fig. 2. Velocity field (arrows) superposed to the contour plots of the full temperature for (a) $\mathrm{Ra}=3 \times 10^{5}$ and $\operatorname{Pr}=0.32007$, (b) $\mathrm{Ra}=10^{5}$ and $\operatorname{Pr}=0.32012$, (c) $\mathrm{Ra}=10^{4}$ and $\operatorname{Pr}=0.32034,(\mathrm{~d}) \mathrm{Ra}=10^{3}$ and $\operatorname{Pr}=0.31983$.

temperature. Depending on the value of Pr these effects are more or less important. Another important effect of decreasing $\mathrm{Pr}$ is the weakening of the boundary layers that also contributes to the transport of hot and cold fluids to the interior of the box in opposite directions.

The effect of decreasing Pr while fixing the value of Ra is much stronger. The change of the dynamics is illustrated in Fig. 3 for $\mathrm{Ra}=10^{4}$. At $\mathrm{Pr}=0.683$ the big vortex is much more confined in the center of the box than for larger Ra, and the boundary layers are wider. At lower Pr two extra vortices appear near the first at top and bottom. This change can be seen in Figs. 3b and 3c. In Fig. 3c the strength of the new vortices is already comparable to the central one, and the formation of two new vortices can be appreciated near the horizontal sides of the slot. Between the vortices the heat transport already affects the interior of the box. At lower Pr the five vortices are fully developed (see Fig. 3d).

The stationary flows examined are center-symmetric, i.e., they satisfy

$$
\psi(t, 1-x, \Gamma-y)=\psi(t, x, y), \quad \Theta(t, 1-x, \Gamma-y)=-\Theta(t, x, y),
$$

which means

$$
u(t, 1-x, \Gamma-y)=-u(t, x, y), \quad v(t, 1-x, \Gamma-y)=-v(t, x, y) .
$$

Concerning to their stability, at $\mathrm{Ra}=10^{3}$ the SSs are stable for any Pr computed. For the other values of Ra, the first four bifurcations found are of Hopf type. The results are summarized in Fig. 1 (the solid lines indicate the stable flows), and in Table 1, where the critical parameters are given. The first transition destabilizes the fluid, i.e. $\operatorname{Pr}_{c}^{1}$ gives the critical Prandtl number, $\operatorname{Pr}_{c}$, where the SSs change from stable to unstable when Pr becomes smaller. Notice that the cusps and loops of the branches at high Ra occur always in their unstable part. According to the table, the flows depicted in Figs. 2a,b and Figs. 3b,c,d are unstable.

The first bifurcation found with $\mathrm{Ra}=10^{4}$ and $\mathrm{Ra}=10^{5}$ does not break the symmetry (7-8), then POs arising at these points keep the symmetry at any time 

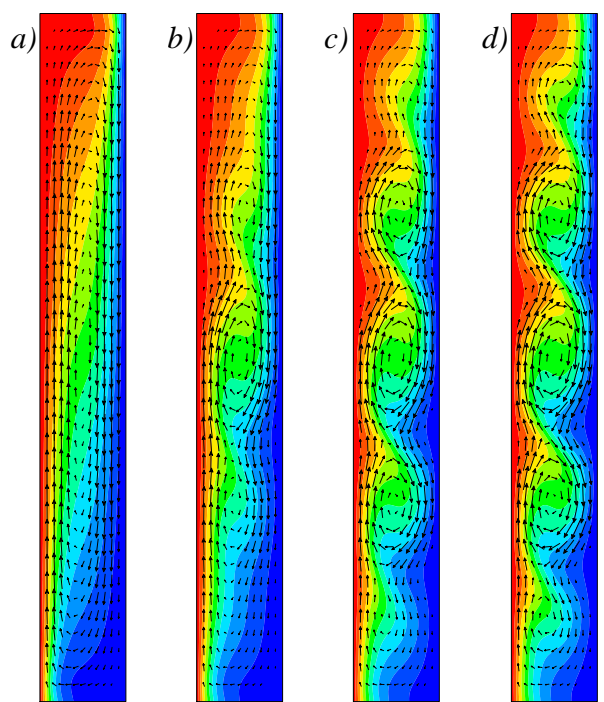

Fig. 3. Idem Fig. 2 for $\mathrm{Ra}=10^{4}$ and (a) $\operatorname{Pr}=0.683$, (b) $\operatorname{Pr}=0.22984$, (c) $\operatorname{Pr}=0.11703$, (d) $\operatorname{Pr}=0.092051$

Table 1. Critical Prandtl numbers, $\operatorname{Pr}_{c}^{i}, i=1, \cdots 4$ and frequencies, $\omega_{c}^{i}$ of the first bifurcations found on the branches of the steady flows in the interval $0.014<\operatorname{Pr}<0.683$ for three values of Ra. The periods are given in diffusion time units.

\begin{tabular}{lcccccccc}
\hline \hline $10^{-4} \mathrm{Ra}$ & $\operatorname{Pr}_{c}^{1}$ & $\omega_{c}^{1}$ & $\operatorname{Pr}_{c}^{2}$ & $\omega_{c}^{2}$ & $\operatorname{Pr}_{c}^{3}$ & $\omega_{c}^{3}$ & $\operatorname{Pr}_{c}^{4}$ & $\omega_{c}^{4}$ \\
\hline 1 & 0.2790 & 27.526 & 0.2622 & 22.425 & 0.1087 & 24.904 & 0.1073 & 24.630 \\
10 & 0.3267 & 350.68 & 0.3245 & 280.51 & 0.3018 & 217.7 & 0.2678 & 169.96 \\
30 & 0.6368 & 764.01 & 0.6314 & 703.81 & 0.6244 & 816.50 & 0.6054 & 636.23 \\
\hline \hline
\end{tabular}

(F-cycles), and the bifurcated POs can destabilize via period doubling bifurcations. However it is broken when $\mathrm{Ra}=3 \times 10^{5}$, but then, they keep the spatio-temporal reflection

$$
\psi(t, 1-x, \Gamma-y)=\psi(t+T / 2, x, y), \quad \Theta(t, 1-x, \Gamma-y)=-\Theta(t+T / 2, x, y),
$$

which means

$$
u(t, 1-x, \Gamma-y)=-u(t+T / 2, x, y), \quad v(t, 1-x, \Gamma-y)=-v(t+T / 2, x, y),
$$

therefore, the orbits are symmetric cycles ( $S$-cycles). For more details see Ref. [17].

To evaluate the physical mechanisms of the instabilities, the contribution of the energies generated by shear, $K_{s}$, and by buoyancy, $K_{b}$, and of the energy dissipated in the fluid, $K_{d}$, to the rate of change of the kinetic energy of the perturbation are computed. They are included in Table 2. As can be seen in the table the beginning of the oscillations is triggered by the shear of the steady flow, and the viscous dissipation almost balance this shear in the three cases. Consequently the energy generated by buoyancy represents a small fraction of the total energy balance at the transition. However, in agreement with Ref. [9], the buoyant kinetic energy is very small and stabilizing when the onset of the oscillations takes place at low Ra and Pr. Notice that since the mean energies of the table depend on a linear problem, only their relative values have physical meaning. By inspecting the contour plots of each of 
Table 2. Total energy generated by shear, $K_{s}$, and by buoyancy, $K_{b}$, and dissipated, $K_{d}$, for the perturbations at the bifurcation points. Notice that at the transition the three terms are in balance.

\begin{tabular}{lcccc}
\hline \hline $10^{-4} \mathrm{Ra}$ & $\operatorname{Pr}_{c}$ & $K_{s}$ & $K_{b}$ & $K_{d}$ \\
\hline 1 & 0.2790 & 1.26032 & -0.03127 & -1.22905 \\
10 & 0.3267 & 8.75613 & 0.12228 & -8.87845 \\
30 & 0.6368 & 20.9519 & 1.05814 & -22.0100 \\
\hline \hline
\end{tabular}

these quantities over the domain it is clear that the perturbations evolve in different locations of the slot. For $\mathrm{Ra}=3 \times 10^{5}, \operatorname{Pr}_{c}=0.6368$, the shear is concentrated near the left upper and the right lower corners of the cavity, while for $\mathrm{Ra}=1 \times 10^{5}, \operatorname{Pr}_{c}=$ 0.3267 it is restricted to a thin strip going from $y \approx \Gamma / 5$ to $y \approx 4 \Gamma / 5$ outside the lateral boundary layers of the basic flow. For $\mathrm{Ra}=1 \times 10^{4}, \operatorname{Pr}_{c}=0.2790$ the shear affects the central part of the fluid. In any case, most of the viscous dissipation is concentrated in the lateral boundary layer in the neighborhood where the production of shear energy takes place. These differences give rise to very different types of oscillations for the distinct parameters of the problem considered.

\section{Periodic orbits and their stability}

As for the SSs, the stable and unstable POs have been computed by Newton-Krylov continuation methods, and their stability by means of the ARPACK package, based on Arnoldi algorithms [19,20]. The first branches of periodic solutions have been continued for the Ra mentioned in Sec.3. Since the bifurcations are supercritical by decreasing Pr, the solutions are stable from the bifurcation point. Each of the subsequent branches (which have not been calculated) has, initially, another Floquet multiplier (FM) or a complex-conjugate pair of them (depending on the type of bifurcation) outside the unit circle more than the preceding.

Table 3. For $i=0$, critical Prandtl number, $\operatorname{Pr}_{c}^{0}$, and frequency $\omega_{c}^{0}$ of the bifurcations where the branches of SSs lose stability. The capital letters $S$ or $F$ beside the corresponding values state if the POs arising from this point are $S$-cycles or $F$-cycles, respectively. For $i=1 \cdots 6$, $\operatorname{Pr}_{c}^{i}$ of the bifurcations, and initial periods, $T^{i}$, of the branches of POs. The symbols $(\bullet / \circ)$ indicate whether the critical multipliers get in or out the unit circle, respectively.

\begin{tabular}{cccc}
\hline \hline$i / \mathrm{Ra}$ & $10^{4}$ & $10^{5}$ & $3 \times 10^{5}$ \\
\hline \hline 0 & $(0.2790,27.526) F$ & $(0.3267,350.68) F$ & $(0.6368,764.01) S$ \\
1 & $(0.2728,0.2552) \circ$ & $(0.3230,0.01774) \circ$ & $(0.5216,0.008430) \circ$ \\
2 & $(0.2676,0.3214) \circ$ & $(0.2972,0.01653) \circ$ & $(0.5168,0.008433) \circ$ \\
3 & $(0.2684,0.3991) \bullet$ & $(0.2734,0.01553) \circ$ & $(0.5047,0.008439) \circ$ \\
4 & $(0.2450,0.4689) \bullet$ & & $(0.4935,0.008442) \circ$ \\
5 & $(0.2383,0.4716) \circ$ & & $(0.4798,0.008441) \circ$ \\
6 & $(0.2022,0.6972) \circ$ & & $(0.4794,0.008440) \circ$ \\
\hline
\end{tabular}

Figure 4a shows a detail of the branch of POs of Fig. 1b, and those bifurcated from it, and Table 3 contains the critical parameters found on the main branch. With $\mathrm{Ra}=10^{4}$ the branch of POs arising from the bifurcation point at $\operatorname{Pr}_{c}^{1}=0.2790$ has a double fold very near the beginning, and turns again towards higher values of Pr at 

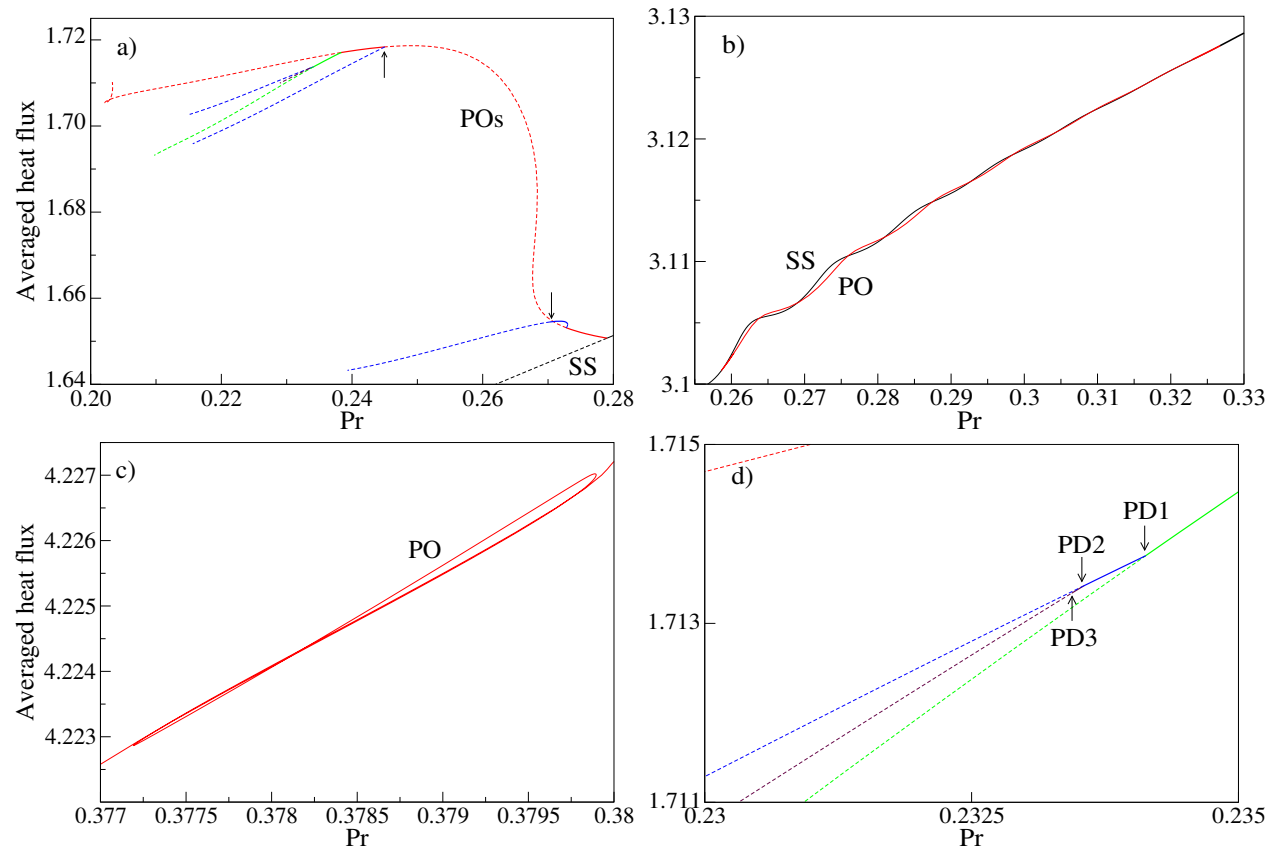

Fig. 4. Blow-ups of Figs. 1b-d showing details of the POs. a) $\mathrm{Ra}=10^{4}$, b) $\mathrm{Ra}=10^{5}$ and c) $\mathrm{Ra}=3 \times 10^{5}$. In a) and d) solid lines mean stable solutions and dashed lines unstable, and online red refers to POs, blue to POs of doubled period, brown to POs of quadrupled period, and green to POs with broken symmetry. d) Detail of the beginning of the period doubling cascade. The arrows in plot a) indicate the position of the POs shown in Figs. 5 and 6 .

0.2022, developing a small loop (in terms of the averaged heat transport). The branch turns again to lower values of Pr at 0.2034. Before the first saddle-node bifurcation there is a period doubling bifurcation of POs at $\operatorname{Pr}=0.2728$ that destabilizes the fluid, and after the second saddle node, there is another period-doubling of POs at $\operatorname{Pr}=0.2450$ that stabilizes the branch, followed by a pitchfork bifurcation of POs at $\operatorname{Pr}=0.2383$ that destabilizes it again. At the third turning point at $\operatorname{Pr}=0.2022$ a second real FM crosses out the unit circle. Both multipliers increase quickly their modulus, and at $\operatorname{Pr}=0.20283826$ they are 2152.4 and 8.2. The period of the orbits increases along the branch. At the lower Pr computed the period of the orbit is four times that at the onset.

Along the principal branch, the $F$-cycles consist of an oscillation of the amplitude of a central and two upper an lower vortices and their merging and splitting. When the vortices are disconnected tongues of hot/cold fluid can penetrate between them to the interior of the slot. The sequence is depicted in Fig. 5 where snapshots along a period of the stable $\mathrm{PO}$ at $\mathrm{Pr}=0.2450$ are plotted. They are not equally-spaced in time in order to capture a detailed description. At the beginning the central vortex loses intensity while the upper and lower gain it. Soon the central vortex regains intensity while all three stretch out and connect forming a global circulation that maintains the hot and cold fluid confined near the lateral sides in most of the box. Later, the long vortex shrinks and the upper and lower vortices start to develop and to increase their strength, closing the cycle.

The first period-doubled branch of POs of Figs. 4a,d, starting at $\operatorname{Pr}=0.2728$, is subcritical, and gains stability in a turning point at $\operatorname{Pr}=0.2730$ to lose it soon 

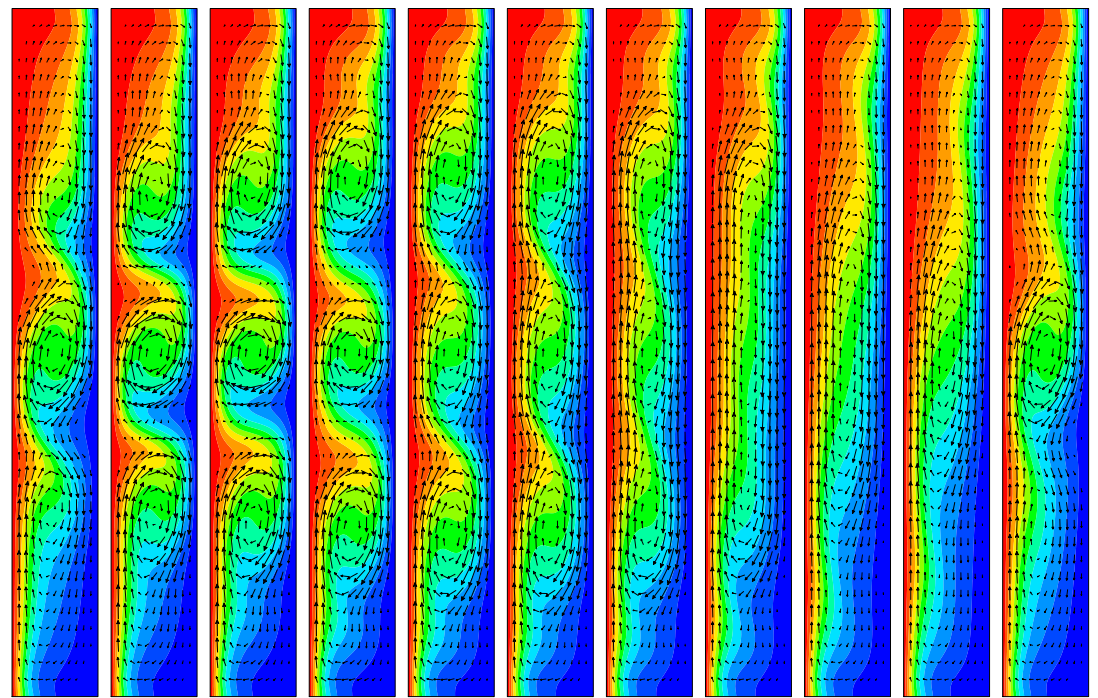

Fig. 5. Snapshots of the velocity field (arrows) superposed to the contour plots of the full temperature in a period of a stable PO. The snapshots are taken at $t=0,2 T / 20$, $3 T / 20,5 T / 20,8 T / 20,10 T / 20,12 T / 20,14 T / 20,16 T / 20,18 T / 20,19 T / 20$. The parameters are $\mathrm{Ra}=10^{4}$ and $\mathrm{Pr}=0.2450$.

at $\operatorname{Pr}=0.2703$ in a Hopf bifurcation. From this point the leading FM increases their modulus quickly. The second period-doubled branch of POs is supercritical, and consequently unstable. It never regains stability since the modulus of the unstable FM soon becomes larger than $10^{4}$. In both period-doubling bifurcations, the critical eigenfunctions are antisymmetric. The POs on these two branches are $S$-cycles (see Eq.(9)). The temporal evolution of one of these orbits is depicted in Fig. 6. There is a connection of the upper and lower vortices with the central one, but now the intensities of the two external vortices alternate, and that of the central scarcely varies, while its center oscillates vertically.

The pitchfork bifurcation gives rise to stable POs without symmetries. However this branch loses stability soon in a new supercritical period doubling bifurcation at $\operatorname{Pr}=0.234139$, and again the unstable FM becomes quickly of modulus much larger than one. The dynamics of the stable POs along a period resembles to that of Fig. 5, with three asymmetric vortices well defined that slowly connect and fastly disconnect. When they are separated the tongues of hot fluid almost penetrate up to the cold boundary layer, and vice versa. The new branch of quaternary flows of doubled period is supercritical, and only stable up to $\operatorname{Pr}=0.233551$, where a new period doubling bifurcation has been localized. The period of the supercritical branch arising from this point is already 1.92 and loses stability at $\operatorname{Pr}=0.233437$. The estimation of the Feigenbaum constant given by the three bifurcation parameters is 5.158. Therefore by decreasing $\mathrm{Pr}$ it would be reasonable to find a period doubling cascade.

For $\mathrm{Ra}=10^{5}$ the steady branch loses stability at $\operatorname{Pr}_{c}^{1}=0.3267$. The new branch of POs is also supercritical, but the period becomes small by decreasing the continuation parameter. It becomes unstable at 0.3230 through a Hopf bifurcation (see Fig. 4b and table 3). The branch of the POs crisscrosses that of the steady solutions in the projection presented. The flow consists of a tiny oscillation of the bulk of the fluid, keeping the structure shown in Fig. 2b. In this case the next two bifurcations found are also of Hopf type, so they give rise to branches of quasi-periodic flows, initially 

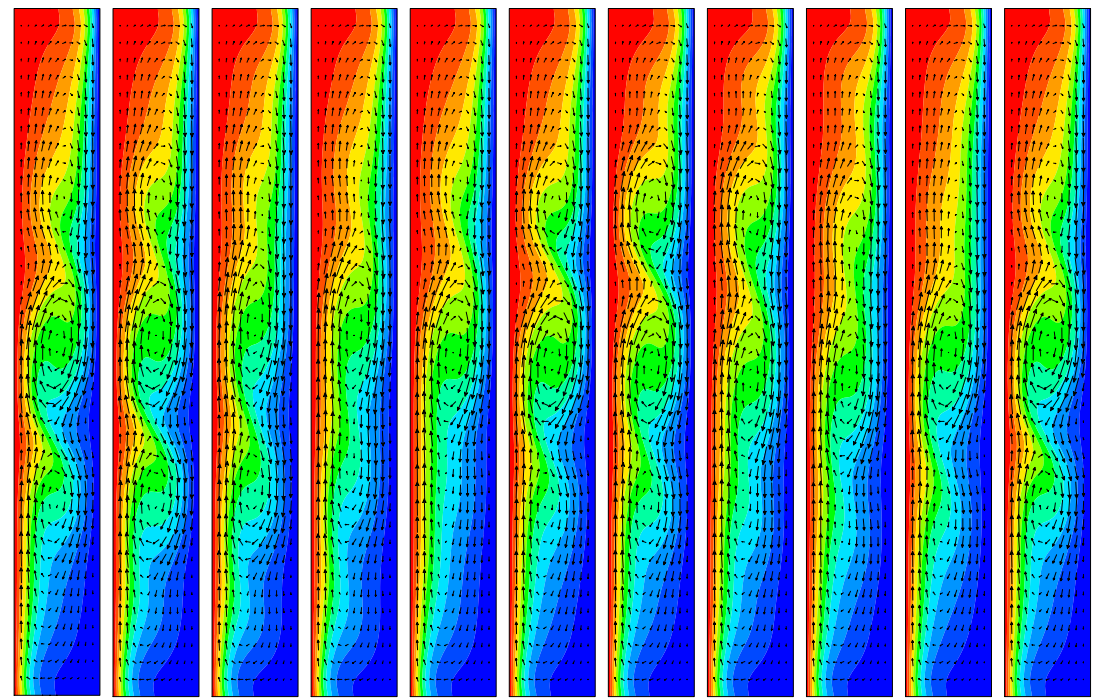

Fig. 6. Snapshots of the velocity field (arrows) superposed to the contour plots of the full temperature in a period of a stable symmetric PO. The snapshots are taken at $t=0, T / 10$, $T / 5,3 T / 10,2 T / 5, T / 2,3 T / 5,7 T / 10,4 T / 15,9 T / 10, T$. The parameters are $\mathrm{Ra}=10^{4}$ and $\operatorname{Pr}=0.2705$.

unstable. The branch arrives up to $\operatorname{Pr}=0.2587$, where it seems that there is a turning point that increases again the averaged heat transport.

For $\mathrm{Ra}=3 \times 10^{5}$, the bifurcation at $\operatorname{Pr}_{c}^{1}=0.6368$ is again supercritical and the period increases up to $T=8.442 \times 10^{-3}$ at $\mathrm{Pr}=0.4879$. Then it starts to decrease. The branch of POs is stable down to $\operatorname{Pr}=0.5216$, and because several eigenvalues cross out the unit circle quickly it seems that it never regains stability. The fifth and sixth bifurcations of Table 3 have almost the same critical parameters, consequently by slightly moving Ra they could collide in a double Hopf bifurcation. The dynamics of the stable flow reminds the dynamics confined near the lateral walls, found in Ref. [17], but at $\operatorname{Pr}=0.5351$ the oscillations hardly affect the boundary layer. There is an almost steady global circulation near the vertical sides of the cavity, and tongues of cold (hot) fluid are transported up (down) by the velocity field near the boundary layer. The only 'anomalies' found on this branch are the laces that appear when the branch of steady solutions becomes wavy. The first of them, shown in Fig. 4c, is between $\operatorname{Pr}=0.3799$ and 0.3772 , and the second between $\operatorname{Pr}=0.3270$ and 0.3238 . The last point we have been able to compute is $\mathrm{Pr}=0.3003$ when a last lace, which starts at $\operatorname{Pr}=0.3091$, turns to high $\operatorname{Pr}$ values.

\section{Conclusions}

It has been found that for a slot of $\Gamma=8$, at large Pr and Ra, the curves of steady solutions are smooth, but at low $\mathrm{Pr}$, always smaller than the critical value where the $S S s$ lose stability, they undulate and develop successive cusps and loops. In this way there are multiple unstable steady solutions at low Pr coming from a single branch of solutions. The stable flows found consist of a single vortex more o less confined depending on Pr. However, in liquids of small Pr and Ra the stable flows develop three vortices. 
The critical Prandtl number $\operatorname{Pr}_{c}$ at which the stationary flows become unstable increases when Ra is increased. The steady flows destabilize via Hopf bifurcations. For any value of Ra considered, the stable POs are confined in a small range of Prandtl numbers. The computation of unstable POs by continuation methods have allowed to find long stable periodic oscillations isolated in small regions of the parameter space at $\operatorname{Pr}$ of order $10^{-1}$, which are very difficult to capture by chance by time integration.

Although in any case the shear is the main component to trigger the oscillations, at high (low) Ra the averaged rate of change of the generation of mechanical energy by the buoyancy force tends to decrease (increase) when Pr decreases in the neighborhood of the bifurcation points, and the type of dynamics of the POs depends mainly on the location of the perturbation. At Pr of order one the instability gives rise to waves traveling because the perturbation is concentrated along the lateral boundary layer. At lower Pr the perturbation affects the body of the fluid and causes the cyclical generation of upper and lower vortices near the central area, and the return to a global circulation. In addition, when $\mathrm{Ra}$ is decreased one order of magnitude the period of the oscillations increases also one order of magnitude.

The results shown in this paper also apply to thermosolutal convection (without Soret effect) with a very large diffusion coefficient. In this case, since the concentration does not affect the stream function and temperature equations, and the concentration diffuses very rapidly, it is not able to destabilize the fluid. Then the dynamics and instabilities expected are those described here. The concentration, driven by the velocity and temperature fields, behaves dynamically as the temperature (see the contour plots) but with opposite sign.

\section{6 acknowledgments}

This work was supported by the Spanish MCYT/FEDER grant FIS2016-76525-P.

All the authors contributed equally to this study.

\section{References}

1. R. Feigelson (Ed.), 50 years Progress in Crystal Growth. A reprint collection, Elsevier, 2004.

2. M. Christon, P. Gresho, S. Sutton, Computational Predictibility of Natural Convection Flows in Enclosures, Int. J. Numer. Meth. Fluids 40 (2002) 953-980.

3. M. Lappa, Thermal Convection: Patterns Evolution and Stability, Wiley, Singapore, 2010.

4. C. K. Mamun, L. S. Tuckerman, Asymmetry and Hopf bifurcation in spherical Couette flow, Phys. Fluids 7 (1995) 80-91.

5. L. S. Tuckerman, D. Barkley, Bifurcation analysis for timesteppers, in: E. Doedel, L. S. Tuckerman (Eds.), Numerical Methods for Bifurcation Problems and Large-Scale Dynamical Systems, Vol. 119 of IMA Volumes in Mathematics and its Applications, Springer-Verlag, 2000, pp. 453-466.

6. K. H. Winters, Oscillatory convection in liquid metals in a horizontal temperature gradient, Int. J. Numer. Meth. Engng 25 (1988) 401-414.

7. A. Y. Gelfgat, P. Z. Bar-Yoseph, A. L. Yarin, Stability of multiple steady states of convection in laterally heated cavities, J. Fluid Mech. 388 (1999) 315-334.

8. S. Xin, P. Le Quéré, Natural-convection flows in air-filled differentially heated cavities with adiabatic horizontal walls, Numer. Heat Transfer. Part A 50 (2006) 437-466.

9. D. Henry, H. BenHadid, Multiple flow transitions in a box heated from the side in low-Prandtl-number fluids, Phys. Rev. E 76 (2007) 016314. 
10. A. Bergeon, E. Knobloch, Periodic and localized states in doubly diffusive convection, Physica D 237 (2008) 1139-1150.

11. J. Sánchez, M. Net, B. García-Archilla, C. Simó, Newton-Krylov continuation of periodic orbits for Navier-Stokes flows, J. Comput. Phys. 201 (1) (2004) 13-33.

12. J. Sánchez, M. Net, B. García-Archilla, C. Simó, Continuation of periodic orbits in largescale dissipative systems, in: F. Dumortier, H. Broer, J. Mawhin, A. Vanderbauwhede, S. V. Lunel (Eds.), Proceedings of the Equadiff-2003 Conference, World Scientific, Singapore, 2005, pp. 625-630.

13. M. Net, J. Sánchez, Continuation of bifurcations of periodic orbits for large-scale systems, SIAM J. Appl. Dynam. Systems 14 (2) (2015) 674-698.

14. S. Wakitani, Flow patterns of natural convection in an air-filled vertical cavity, Phys. Fluids 10 (8) (1998) 1924-1928.

15. H. Yahata, Stability analysis of natural convection in vertical cavities with lateral heating, J. Phys. Soc. Jpn. 66 (11) (1998) 3434-3443.

16. S. Xin, P. Le Quéré, Stability of two-dimensional (2D) natural convection flows in airfilled differentially heated cavities: 2D/3D disturbances, Fluid Dynamics Research 44 (3) (2012) 031419.

17. M. Net, J. Sánchez, Periodic orbits in tall laterally heated rectangular cavities, Phys. Rev. E 95 (2017) 023102.

18. H. Ke, Y. He, Y. Liu, F. Cui, Mixture working gases in thermoacoustic engines for different applications, Int. J. Thermophys. 33 (2012) 1143-1163.

19. J. Sánchez, M. Net, Numerical continuation methods for large-scale dissipative dynamical systems, Eur. Phys. J. Spec. Top. 225 (13) (2016) 2465-2486.

20. R. B. Lehoucq, D. C. Sorensen, Deflation techniques for an implicitly restarted Arnoldi iteration, SIAM J. Matrix Anal. Appl. 17 (1996) 789-821. 